\title{
Central polynomials for matrix algebras over the Grassmann algebra
}

\author{
S. M. Alves Jorge \\ Departamento de Matemática, Pontifícia Universidade Católica de Minas Gerais - \\ 30535-610 - Belo Horizonte - Brazil \\ E-mail address: sandramara@pucminas.br
}

\author{
A. C. Vieira \\ Departamento de Matemática, Instituto de Ciências Exatas, Universidade Federal de \\ Minas Gerais, 31123-970 - Belo Horizonte - Brazil \\ E-mail address: anacris@ufmg.br
}

\begin{abstract}
In this work, we describe a method to construct central polynomials for $F$-algebras where $F$ is a field of characteristic zero. The main application deals with the $T$-prime algebras $M_{n}(E)$, where $E$ is the infinite- dimensional Grassmann algebra over $F$, which play a fundamental role in the theory of PI-algebras. The method is based on the explicit decomposition of the group algebra $F S_{n}$.

AMS Classification 2000: Primary 16R10, Secondary 16W50, 15A75.

Keywords: Polynomial identities, central polynomials, Grassmann algebra.
\end{abstract}

\section{Introduction}

Let $F$ be a field of characteristic zero. The set of polynomial identities satisfied by a $F$-algebra $A$, denoted by $\operatorname{Id}(A)$, is a $T$-ideal of the free algebra $F\langle X\rangle$ of polinomials over $F$, i.e., $\operatorname{Id}(A)$ is invariant over all endomorphisms of $F\langle X\rangle$. One of the main goals of the theory of PI-algebras, which are algebras satisfying non-trivial polynomial identities, is to determine their $T$-ideals. For each one of the $T$-prime algebras, classified by Kemer in [7], the $T$-ideal has been insistently studied and there are few known results. For example, we have the complete description just for $\operatorname{Id}(E), \operatorname{Id}\left(M_{2}(F)\right)$ and $\operatorname{Id}\left(M_{1,1}(E)\right)$ (see [9], [2] and [11], respectively). Remember that Kemer showed that the only non-trivial $T$-prime algebras in characteristic zero are 
the full matrix algebra $M_{k}(F)$, the algebra $M_{k}(E)$ of matrices over the the Grassmann algebra $E$ and $M_{r, s}(E)$, a particular subalgebra of $M_{r+s}(E)$.

Trying to get new identities for an algebra $A$ we can consider the set $C(A)$ of central polynomials of $A$ which consists of $f\left(x_{1}, \cdots, x_{n}\right) \in F\langle X\rangle$ such that $f\left(a_{1}, \cdots, a_{n}\right)$ belongs to the center of $A, \forall a_{1}, \cdots, a_{n} \in A$. The existence of central polynomails is of great interest not only for the development of the structure theory of PI-algebras, but also for the combinatorial PI-theory (as a reference, see[4]). Since $\operatorname{Id}(A) \subset C(A)$ it is interesting to construct central polynomials which are non identities of $A$, called nontrivial central polynomials. Once we have a non-trivial central polynomial $f=f\left(x_{1}, \cdots, x_{n}\right)$ of degree $k$ of $A$ we get a new polynomial from the commutator $[f, x]$ of degree $k+1$ which belongs to $\operatorname{Id}(A)$.

For the $T$-prime algebras, the existence of non-trivial central polynomials was proved by Kemer in [8] but in spite of the importance of the these algebras, the concrete form of the set of their central polynomials is far from being known, the only case which is completely settled is that of $M_{2}(F)$ (see [10]). Several attempts of getting new results have been considered. For example, in [1], Bondari developed a computational method to find all central polynomials of degree less than 9 for $M_{3}(F)$. In [3], V. Drensky constructed an element in $C\left(M_{k}(F)\right) \backslash I d\left(M_{k}(F)\right)$ of degree $(k-1)^{2}+4$ for any $k \geq 3$ and it is the minimal degree known for a non-trivial central polynomial of $M_{k}(F)$ until this moment. Non-trivial central polynomials for the algebras $M_{k, l}(E)$ were constructed by Razmyslov in [12].

In this work we present a method to construct elements in $C\left(M_{k}(E)\right) \backslash$ $\operatorname{Id}\left(M_{k}(E)\right)$ based on the explicited decomposition of the group algebra $F S_{k}$. The technics developed here were described from an algorithmic process which was implemented by using the free software GAP [5] for the case $k=2$. We were able to show that the minimal degree of a non-trivial central polynomial in $M_{2}(E)$ is $\geq 9$.

\section{The algebra $F S_{n}$, PI-algebras and central polynomials}

The representation theory of the symmetric group $S_{n}$ over a field of characteristic zero is a very useful tool in the developing of the theory of PI-algebras. We start this section with some basic facts on this topic.

By fixing a partition $\lambda=\left(\lambda_{1}, \ldots, \lambda_{h}\right)$ of $n$, we associate to it the Young diagram $D_{\lambda}$ which consists of $n$ boxes $\square$ in the following way

We observe that the lengths of the columns form a new partition of $n$, $\lambda^{\prime}:=\left(\lambda_{1}^{\prime}, \ldots, \lambda_{r}^{\prime}\right)$, where $\lambda_{i}^{\prime}=\sum_{\substack{\nu \\ \lambda_{\nu} \geq i}} 1$, called conjugate partition of $\lambda$. 


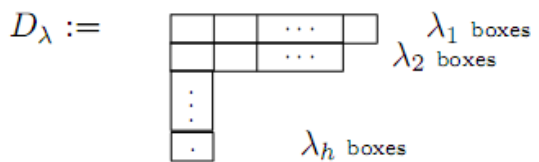

A Young tableau $T_{\lambda}$ of a diagram $D_{\lambda}$ is a filling of boxes of $D_{\lambda}$ with the integers $1,2, \ldots, n$. We say that $T_{\lambda}$ is a standard Young tableau of shape $\lambda$ if the integers in each row and in each column increase from left to right and from top to bottom, respectively. For example, for the partition $\lambda=(2,1)$ of $n=3$ we have 6 Young tableaux of shape $\lambda$ and only two of them are standard:

$$
\begin{array}{|l|l|l|l|}
\hline 1 & 2 \\
\hline 3 &
\end{array} \text { and } \begin{array}{|l|l|}
\hline 1 & 3 \\
\hline 2 &
\end{array}
$$

In general, the number of standard Young tableaux of shape $\lambda$ is given by the hook formula (see [6]). During this text we consider $d_{\lambda}$ the number of Young standard tableau of shape $\lambda$ and the set $\left\{T_{1}, T_{2}, \cdots, T_{d_{\lambda}}\right\}$ of such tableaux in lexicographic order, that is, $T_{1}<T_{2}<\cdots<T_{d_{\lambda}}$.

The row-stabilizer of a Young tableau $T_{\lambda}$ is defined as

$$
R_{T_{\lambda}}=S_{\lambda_{1}}\left(a_{11}, \cdots, a_{1 \lambda_{1}}\right) \times \ldots \times S_{\lambda_{h}}\left(a_{h 1}, \cdots, a_{h \lambda_{h}}\right)
$$

where $S_{\lambda_{i}}\left(a_{i 1}, \cdots, a_{i \lambda_{i}}\right)$ denotes the symmetric group on the integers $a_{i 1}, \cdots, a_{i \lambda_{i}}$. Analogously, the column-stabilizer of $T_{\lambda}$ is

$$
C_{T_{\lambda}}=S_{\lambda_{1}^{\prime}}\left(a_{11}, \cdots, a_{\lambda_{1}^{\prime} 1}\right) \times \ldots \times S_{\lambda_{r}^{\prime}}\left(a_{1 \lambda_{1}}, \cdots, a_{\lambda_{r}^{\prime} \lambda_{1}}\right)
$$

where $\lambda^{\prime}=\left(\lambda_{1}^{\prime}, \cdots, \lambda_{r}^{\prime}\right)$ is the conjugate partition of $\lambda$.

Furthermore, we define the essential idempotent associated to $T_{\lambda}$ by

$$
e_{T_{\lambda}}:=\sum_{\rho \in R_{T_{\lambda}}} \sum_{\sigma \in C_{T_{\lambda}}}(\operatorname{sgn} \sigma) \rho \sigma .
$$

We will use $e_{i}$ (or $e_{i}^{\lambda}$ if necessary) to denote the essential idempotent $e_{T_{i}}$. In general, the $e_{j}^{\prime} s$ are not orthogonal but we have that $e_{j} e_{i}=0$, when $T_{i}<T_{j}$.

We observe that $e_{\gamma T_{\lambda}}=\gamma e_{T_{\lambda}} \gamma^{-1}$ for all $\gamma \in S_{n}$. Moreover it is possible to prove that there exists a non-zero integer $q$ such that $e_{T_{\lambda}}^{2}=q e_{T_{\lambda}}$ for all Young tableaux $T_{\lambda}$; it follows that the element $e=\frac{1}{q} e_{T_{\lambda}} \in F S_{n}$ is idempotent. As an important property of the essential idempotents, we have that the left $F S_{n}$-modules $F S_{n} e_{T_{\lambda}}$ and $F S_{n} e_{\widetilde{T}_{\lambda}}$ are isomorphic when $T_{\lambda}$ and $\widetilde{T}_{\lambda}$ are Young tableaux of same shape $\lambda$. Whereas $T_{\lambda}$ and $T_{\mu}$ are of different shape, the modules $F S_{n} e_{T_{\lambda}}$ and $F S_{n} e_{T_{\mu}}$ are not isomorphic. The next theorem shows that the standard Young tableaux come into play if one 
wants to find, amoung the $n$ ! essential idempotents arising from tableaux of shape $\lambda$, some orthogonal ones (see [6]).

Theorem 2.1. We have

$$
F S_{n}=\bigoplus_{\lambda \vdash n} I_{\lambda} \text {, with } I_{\lambda}=\bigoplus_{T_{\lambda} \text { standard }} F S_{n} e_{T_{\lambda}}
$$

where $I_{\lambda}$ is a two-sided ideal with $\operatorname{dim} I_{\lambda}=d_{\lambda}^{2}$. Moreover, each left module $F S_{n} e_{T_{\lambda}}$ is minimal and $\operatorname{dim} F S_{n} e_{T_{\lambda}}=d_{\lambda}$.

It follows from the theorem above that $e_{T_{\lambda}} e_{T_{\mu}}=0$, when $\lambda$ and $\mu$ are different partitions of $n$.

By considering $\lambda \vdash n$ a fixed partition, we denote by $S_{i j}$ (or $S_{i j}^{\lambda}$ ) the permutation of $S_{n}$ which takes the tableau $T_{j}$ to the tableau $T_{i}$, that is, $S_{i j} T_{j}=T_{i}$. Obviously, $S_{i j}^{-1}=S_{j i}$. And as we have observed above, it follows that $S_{i j} e_{j}=e_{i} S_{i j}$ and the following is true.

Lemma 2.2. By a fixed partition $\lambda$ of $n$, we have

(1) For each $k \in\left\{1, \cdots, d_{\lambda}\right\}$, the set $\left\{e_{i} S_{i k}\right\}_{i=1}^{d_{\lambda}}$ is a $F$-basis of $F S_{n} e_{k}$.

(2) The set $\left\{e_{i} S_{i j}\right\}_{i, j=1}^{d_{\lambda}}$ is a F-basis of $I_{\lambda}$.

Proof. Since $e_{i} S_{i k}=S_{i k} e_{k}$ for a fixed $k$, we have $J_{i}=\operatorname{span}\left\{e_{i} S_{i k}\right\}$ is a unidimensional space of $F S_{n} e_{k}$ for all $i=1, \cdots, d_{\lambda}$. As we have observed, $e_{j} e_{i}=0$ when $T_{i}<T_{j}$ in the set $\left\{T_{1}, T_{2}, \cdots, T_{d_{\lambda}}\right\}$ of standard Young tableaux of shape $\lambda$; thus the sum $J_{1}+\cdots+J_{d_{\lambda}}$ is direct. So, $\operatorname{dim}\left(J_{1} \oplus\right.$ $\left.\cdots \oplus J_{d_{\lambda}}\right)=d_{\lambda}$ and then $J_{1} \oplus \cdots \oplus J_{d_{\lambda}}=F S_{n} e_{k}$, from Theorem 2.1.

Now since

$$
I_{\lambda}=\bigoplus_{T_{\lambda} \text { standard }} F S_{n} e_{T_{\lambda}}
$$

we have the set $\left\{e_{i} S_{i j}\right\}_{i, j=1}^{d_{\lambda}}$ generates $I_{\lambda}$ and using Theorem 2.1, we get $\operatorname{dim} I_{\lambda}=d_{\lambda}^{2}$. Then $\left\{e_{i} S_{i j}\right\}_{i, j=1}^{d_{\lambda}}$ forms a basis of $I_{\lambda}$.

Now, if a $F$-algebra $A$ satisfies a non trivial polynomial identity $f\left(x_{1}, \cdots, x_{n}\right) \in F\langle X\rangle$, i.e. $f\left(a_{1}, \cdots, a_{n}\right)=0$, for all $a_{1}, \cdots, a_{n} \in A$ then denote it by $f \equiv 0$ in $A$ and say that $A$ is a PI-algebra. In characteristic zero, it is well known that the ideal $\operatorname{Id}(A)$ of identities satisfied by $A$ is finitely generated by its multilinear ones (which are linear in each of its variables) so we consider the $F$-space of multilinear polynomials in the first 
$n$ variables $x_{1}, \cdots, x_{n}$, that is, $P_{n}=\operatorname{span}_{F}\left\{x_{\sigma(1)} \cdots x_{\sigma(n)} \mid \sigma \in S_{n}\right\}$ and define the following left action of $S_{n}$ over $P_{n}$

$$
\sigma f\left(x_{1}, \cdots, x_{n}\right)=f\left(x_{\sigma(1)}, \cdots, x_{\sigma(n)}\right),
$$

where $\sigma \in S_{n}$. We observe that $F S_{n}$ and $P_{n}$ are isomorphic (as $F$-vector spaces) from the linear isomorphism $\psi: F S_{n} \rightarrow P_{n}$ given by

$$
\psi(\sigma)=x_{\sigma^{-1}(1)} x_{\sigma^{-1}(2)} \cdots x_{\sigma^{-1}(n)} .
$$

Recording that $f\left(x_{1}, \cdots, x_{n}\right)$ is a central polynomial of $A$ if $f\left(a_{1}, \cdots, a_{n}\right)$ belongs to the center of $A$ for all $a_{1}, \cdots, a_{n} \in A$, it is well known that the set

$$
C(A)=\{f \in F\langle X\rangle \mid[f, x] \in \operatorname{Id}(A)\}
$$

formed by all central polynomials of $A$ is generated, as a $T$-space, by its multilinear polynomials.

In this work, we are interested in constructing polynomials in $C(A) \cap P_{n}$ which are not identities of $A$ for a particular algebra $A$. Then by using the isomorphism $\psi$, we have to take elements $\alpha \in F S_{n}$ such that $\psi(\alpha) \in$ $C(A) \backslash \operatorname{Id}(A) \cap P_{n}$.

Definition 2.3. We say that $\alpha \in F S_{n}$ is an element (or multilinear element) of degree $n$ of $A$ if and only if $\psi(\alpha) \in C(A)$.

We observe the following fact.

Lemma 2.4. (1) If $\alpha \in F S_{n}$ is an element of degree $n$ of $A$ then $\alpha . \beta$ is also an element of degree $n$ of $A$ for any $\beta \in F S_{n}$.

(2) If $\lambda$ is a partition of $n$ and $g_{\lambda} \in I_{\lambda}$ is such that $g_{\lambda}=g_{\lambda}^{1}+g_{\lambda}^{2}+\cdots+$ $g_{\lambda}^{d_{\lambda}} \in I_{\lambda}$ where $g_{\lambda}^{k} \in F S_{n} e_{k}$, for all $k=1, \cdots, d_{\lambda}$ and $q$ is the non zero integer such that $e_{k}^{2}=q e_{k}$, then we have

(a) $g_{\lambda}^{k}\left(\frac{1}{q} e_{k} S_{k k}\right)=g_{\lambda}^{k}$ for all $k=1, \cdots, d_{\lambda}$.

(b) $g_{\lambda}^{k}\left(\frac{1}{q} e_{r} S_{r r}\right)=0$ if $k>r$.

(c) Recursively defining $\theta_{k}^{\lambda}$ by $\theta_{1}^{\lambda}=\frac{1}{q} e_{1} S_{11}$ and $\theta_{k}^{\lambda}=\left(1-\theta_{1}^{\lambda}-\theta_{2}^{\lambda}-\cdots-\theta_{k-1}^{\lambda}\right) \frac{1}{q} e_{k} S_{k k}$, for all $k=2, \cdots, d_{\lambda}$ we have

(i) $g_{\lambda} \cdot \theta_{k}^{\lambda}=g_{\lambda}^{k}$, for all $k=1, \cdots, d_{\lambda}$.

(ii) $g_{\mu} \cdot \theta_{k}^{\lambda}=0$, for all partition $\mu$ of $n$ different from $\lambda$.

Proof. To show the first item, let $\alpha=\sum \alpha_{\sigma} \sigma, \beta=\sum \beta_{\tau} \tau$ be elements of $F S_{n}$ and for each $\tau$, consider the endomorphism $\varphi_{\tau}$ of $F\langle X\rangle$ such that $x_{i} \mapsto x_{\tau^{-1}(i)}$ for all $i=1, \cdots, n$ and fixes all the remaining variables. Thus 
for each $\tau \in S_{n}$ we have

$$
\begin{aligned}
\psi(\alpha . \tau) & =\psi\left(\left(\sum \alpha_{\sigma} \sigma\right) \cdot \tau\right)=\sum \alpha_{\sigma} \psi(\sigma \cdot \tau) \\
& =\sum \alpha_{\sigma} x_{\tau^{-1}\left(\sigma^{-1}(1)\right)} x_{\tau^{-1}\left(\sigma^{-1}(2)\right)} \cdots x_{\tau^{-1}\left(\sigma^{-1}(n)\right)} \\
& =\sum \alpha_{\sigma} \varphi_{\tau}\left(x_{\sigma^{-1}(1)} x_{\sigma^{-1}(2)} \cdots x_{\sigma^{-1}(n)}\right) \\
& =\sum \alpha_{\sigma} \varphi_{\tau}(\psi(\sigma))=\varphi_{\tau}(\psi(\alpha))
\end{aligned}
$$

and for a fixed variable $x$,

$$
\begin{aligned}
{[\psi(\alpha . \beta), x] } & =\left[\psi\left(\alpha \cdot\left(\sum \beta_{\tau} \tau\right)\right), x\right] \\
& =\sum \beta_{\tau}[\psi(\alpha \cdot \tau), x] \\
& =\sum \beta_{\tau}\left[\varphi_{\tau}(\psi(\alpha)), \varphi_{\tau}(x)\right] \\
& =\sum \beta_{\tau} \varphi_{\tau}([\psi(\alpha), x]) .
\end{aligned}
$$

It follows that if $\psi(\alpha) \in C(A)$, i.e., $[\psi(\alpha), x] \in I d(A)$ then $\psi(\alpha . \beta) \in C(A)$.

Now we fix a partition $\lambda$ and consider $g_{\lambda}=g_{\lambda}^{1}+g_{\lambda}^{2}+\cdots+g_{\lambda}^{d_{\lambda}} \in I_{\lambda}$ such that $g_{\lambda}^{k} \in F S_{n} e_{k}$, for all $k=1, \cdots, d_{\lambda}$. For a fixed $k \in\left\{1, \cdots, d_{\lambda}\right\}$, since $\left\{e_{i} S_{i k}\right\}_{i=1}^{d_{\lambda}}$ is a basis for $F S_{n} e_{k}$ it follows that there exist $\alpha_{i}$ 's in $F$ such that $g_{\lambda}^{k}=\sum_{i=1}^{d_{\lambda}} \alpha_{i} e_{i} S_{i k}$. On the other hand for all $i=1, \cdots, d_{\lambda}$ we have

$$
e_{i} S_{i k}\left(\frac{1}{q} e_{k} S_{k k}\right)=\frac{1}{q} e_{i} e_{i} S_{i k} S_{k k}=\frac{1}{q} e_{i}^{2} S_{i k}=\frac{1}{q} q e_{i} S_{i k}=e_{i} S_{i k}
$$

that implies $g_{\lambda}^{k}\left(\frac{1}{q} e_{k} S_{k k}\right)=g_{\lambda}^{k}$. Moreover if $k>r$ then

$$
e_{i} S_{i k}\left(\frac{1}{q} e_{r} S_{r r}\right)=\frac{1}{q} S_{i k} e_{k} e_{r} S_{r r}=0 .
$$

In this way we have proved 2.(a) and 2.(b). To see item 2.(c).ii, it is enough to note that $g_{\mu} \in I_{\mu}$ and $\theta_{k}^{\lambda} \in I_{\lambda}$ so that $I_{\mu} I_{\lambda}=\{0\}$ if $\mu$ and $\lambda$ are different partitions of $n$. The item 2.(c).i. can be proved by induction on $k$ and we are done.

The Theorem 2.1 and last lemma show that we can write each element $g$ of degree $n$ of $A$ as a sum

$$
g=\bigoplus_{\lambda \vdash n} g_{\lambda}
$$

where $g_{\lambda}=g_{\lambda}^{1}+g_{\lambda}^{2}+\cdots+g_{\lambda}^{d_{\lambda}} \in I_{\lambda}$ and $g_{\lambda}^{k} \in F S_{n} e_{k}$ is also an element of degree $n$ of $A$, for each partition $\lambda$ of $n$. In fact, from item 1 of Lemma 2.4 , we have that $g .\left(\theta_{1}^{\lambda}+\theta_{2}^{\lambda}+\cdots+\theta_{d_{\lambda}}^{\lambda}\right)$ is an element of degree $n$ of $A$. By using item 2.(c), we have

$g \cdot\left(\theta_{1}^{\lambda}+\theta_{2}^{\lambda}+\cdots+\theta_{d_{\lambda}}^{\lambda}\right)=g_{\lambda} \cdot\left(\theta_{1}^{\lambda}+\theta_{2}^{\lambda}+\cdots+\theta_{d_{\lambda}}^{\lambda}\right)=g_{\lambda}^{1}+g_{\lambda}^{2}+\cdots+g_{\lambda}^{d_{\lambda}}=g_{\lambda}$. 
Furthermore, since $g_{\lambda}=g_{\lambda}^{1}+g_{\lambda}^{2}+\cdots+g_{\lambda}^{d_{\lambda}}$ where $d_{\lambda}$ is the number of standard Young tableaux of shape $\lambda$ and $g_{\lambda}^{k} \in F S_{n} e_{k}$, it follows from items 1 and 2.(c) of the last lemma that $g_{\lambda}^{k}$ is also an element of degree $n$ of $A$.

Thus

$$
\begin{gathered}
g_{\lambda} \text { is an element of degree } n \text { of } A \Leftrightarrow \\
\psi\left(g_{\lambda}\right)=\psi\left(g_{\lambda}^{1}\right)+\psi\left(g_{\lambda}^{2}\right)+\cdots+\psi\left(g_{\lambda}^{d_{\lambda}}\right) \in \psi\left(I_{\lambda}\right) \cap C(A) \Leftrightarrow \\
\psi\left(g_{\lambda}^{k}\right) \in \psi\left(F S_{n} e_{T_{\lambda}}\right) \cap C(A) \text {, for all } k=1, \cdots, d_{\lambda} \Leftrightarrow \\
g_{\lambda}^{k} \text { is an element of degree } n \text { of } A, \text { for all } k=1, \cdots, d_{\lambda} .
\end{gathered}
$$

In this way, by fixing a partition $\lambda$ of $n$ and taking $d=d_{\lambda}$; for each $k=1, \cdots, d$, we will use that $\left\{e_{i} S_{i k}\right\}_{i=1}^{d}$ is abasis of $F S_{n} e_{k}$, and construct just the elements of degree $n$ of $A$. In particular, we are interested in elements of type

$$
\alpha_{1} e_{1} S_{1 k}+\alpha_{2} e_{2} S_{2 k}+\cdots+\alpha_{d} e_{d} S_{d k}, \alpha_{i} \in F .
$$

Note that for each partition $\lambda \vdash n$, we can consider $T_{1}$ the standard Young tableau which the columns, from the first to the last one, were filling from the top to the bottom in increasing order with $1, \cdots, n$ and we call it canonical tableau.

Now the isomorphism between $F S_{n} e_{1}$ and $F S_{n} e_{k}$ can be given by $\gamma \mapsto$ $\gamma S_{1 k}$ and so for an element of degree $n$ of $A$

$$
f=\alpha_{1} e_{1} S_{11}+\alpha_{2} e_{2} S_{21}+\cdots+\alpha_{d} e_{d} S_{d 1}
$$

in $F S_{n} e_{1}$ we have, from item 1 of Lemma 2.4, an element of degree $n$ of $A$

$$
f S_{1 k}=\alpha_{1} e_{1} S_{1 k}+\alpha_{2} e_{2} S_{2 k}+\cdots+\alpha_{d} e_{d} S_{d k}
$$

in $F S_{n} e_{k}$ and vice-versa.

Definition 2.5. The elements of degree $n$ which are linear combinations of $e_{i} S_{i 1}$, with $i=1, \cdots, d_{\lambda}$ are called elements of type $\mathcal{T}$ of $A$.

We conclude that in order to determine the multilinear elements of degree $n$ of $A$ it is enough to consider, for each partition $\lambda$ of $n$, elements of type $\mathcal{T}$ of $A$ and so we have proved the next result.

Theorem 2.6. The elements of degree $n$ of $A$ for a fixed partition $\lambda \vdash n$ are linear combinations over $F$ of elements of type $\mathcal{T}$

$$
\sum_{i=1}^{d} \alpha_{i} e_{i} S_{i 1},
$$

where $\alpha_{i} \in F$, for all $i=1, \cdots, d$ and $d$ is the number of standard Young tableaux of shape $\lambda$. 


\section{Elements of type $\mathcal{T}$ in $M_{2}(F)$ and central polynomials of $M_{2}(E)$}

We consider the $T$-prime algebra $M_{2}(E)$, where $E$ is the Grassmann algebra of infinite dimension over the field $F$ generated by $\left\{1, v_{1}, v_{2}, \cdots \mid v_{i} v_{j}=\right.$ $-v_{j} v_{i}$ \} and want to apply the method developed in the last section to determine elements of type $\mathcal{T}$ of a specifical degree $n$ which are non-trivial central polynomials of $M_{2}(E)$. In order to do it, for each partition $\lambda$ of $n$, we inicially construct the elements of type $\mathcal{T}$ in $C\left(M_{2}(F)\right)$. Since the minimal degree of an identity of $M_{2}(E)$ is 8 (see [13]), it is natural to start the calculations with $n=7$.

The arguments used in this work produce a systematic process and now we describe how we constructed computational routines (CR) which were implemented in the software GAP [5] in order to determine central polynomials of $M_{2}(F)$.

CR "CentralCoeffTest $(n)$ " : We know that if $f=f\left(x_{1}, \cdots, x_{n}\right)$ is an element of degree $n$ of $A$ of type $\mathcal{T}$ then there exist $\alpha_{1}, \ldots, \alpha_{d} \in F$ such that

$$
f=\sum_{k=1}^{d} \alpha_{k} S_{k 1} e_{1}=\sum_{k=1}^{d} \alpha_{k} F_{k}\left(x_{1}, \cdots, x_{n}\right) .
$$

On the other hand, for each $k=1, \ldots, d$,

$$
F_{k}=F_{k}\left(x_{1}, \cdots, x_{n}\right)=\sum_{p \in R_{T_{1}}} \sum_{q \in C_{T_{1}}}(\operatorname{sgn} q) S_{k 1} p q
$$

and from the isomorphism $\psi$ we have

$$
F_{k}=\sum_{p \in R_{T_{1}}} \sum_{q \in C_{T_{1}}}(\operatorname{sgn} q) x_{\left(S_{k 1} p q\right)^{-1}(1)} x_{\left(S_{k 1} p q\right)^{-1}(2)} \cdots x_{\left(S_{k 1} p q\right)^{-1}(n)}
$$

where $T_{1}$ is the canonical Young tableau of shape $\lambda$. We observe that to determine $F_{k}$ it is enough to construct $R_{T_{1}}, C_{T_{1}}$ and $S_{k 1}$. Since we work with multilinear polynomials, in the substitutions it is enough to consider the elementary matrices $E_{i j}$, that is, whose entries are equal to 0 except the $(i, j)$ entry which is 1 .

Now we have to find $\alpha_{k}^{\prime} s$ such that $\left[f, x_{n+1}\right] \equiv 0$ in $M_{2}(F)$, that is

$$
\sum_{k=1}^{d} \alpha_{k}\left[F_{k}\left(x_{1}, \cdots, x_{n}\right), x_{n+1}\right] \equiv 0 \text { in } M_{2}(F) .
$$


In this way, for any set $\left\{A_{1}, \cdots, A_{n+1}\right\}$ of elementary matrices of $M_{2}(F)$ we have

$$
\sum_{k=1}^{d} \alpha_{k}\left[F_{k}\left(A_{1}, \cdots, A_{n}\right), A_{n+1}\right]=0 .
$$

By considering for each $k$

$$
\left[F_{k}\left(A_{1}, \cdots, A_{n}\right), A_{n+1}\right]=\left(\begin{array}{cc}
x_{k} & y_{k} \\
z_{k} & w_{k}
\end{array}\right) \in M_{2}(\mathbb{Q}) \subseteq M_{2}(F)
$$

and replacing it in the equality above, we get

$$
\sum_{k=1}^{d} \alpha_{k}\left(\begin{array}{cc}
x_{k} & y_{k} \\
z_{k} & w_{k}
\end{array}\right)=0 \Leftrightarrow \underbrace{\left(\begin{array}{ccc}
x_{1} & \cdots & x_{d} \\
y_{1} & \cdots & y_{d} \\
z_{1} & \cdots & z_{d} \\
w_{1} & \cdots & w_{d}
\end{array}\right)}_{C} \underbrace{\left(\begin{array}{c}
\alpha_{1} \\
\alpha_{2} \\
\vdots \\
\alpha_{d}
\end{array}\right)}_{X}=\left(\begin{array}{c}
0 \\
0 \\
\vdots \\
0
\end{array}\right) .
$$

- We have $4^{n+1}$ possibilities to choose the elementary matrices $E_{11}, E_{12}$, $E_{21}, E_{22}$ to form a colection of $n+1$ matrices.

- After testing all of the possibilities we can form a system $B X=0$ where $B$ is the reduced echelon form of the matrix formed by the aglutination of matrices $C$ in each possibility, having $4^{n+2}$ rows and $d$ columns.

- The next step is solving the system.

- A new routine "ProbablePolynomials $(B, n)$ " describes the possible polynomials to be $f$. This routine uses other routine, called "MonomialTest (Partition)", to determine the polynomials $F_{1}, F_{2}, \cdots, F_{d}$ which works in the following way:

* If the rank of $B$ is less than $d$ we have nonzero values for $\alpha_{k}$ 's which will form a list of candidates different from zero.

* If the rank of $B$ is $d$ we have $\alpha_{1}=\alpha_{2}=\cdots=\alpha_{d}=0$ and so $f=0$. It means that is there is no element different from zero of degree $n$ of $M_{2}(F)$ for the partition $\lambda$.

* Finally, if $B=0$ then for each one of $4^{n+1}$ possibilities, the matrix

$$
\left[F_{k}\left(A_{1}, A_{2}, \cdots, A_{n}\right), A_{n+1}\right]=\left(\begin{array}{cc}
x_{k} & y_{k} \\
z_{k} & w_{k}
\end{array}\right)
$$


is null, for all $k=1,2, \cdots, d$. Thus for all $k=1,2, \cdots, d$, we have that

$$
\left[F_{k}\left(A_{1}, A_{2}, \cdots, A_{n}\right), A_{n+1}\right] \equiv 0 \text { in } M_{2}(F)
$$

and so the polynomials $F_{1}, \cdots, F_{d}$ are elements of degree $n$ of type $\mathcal{T}$ of $M_{2}(F)$.

The next result guarantees that the elements of type $\mathcal{T}$ of $M_{2}(E)$ comes from the elements of type $\mathcal{T}$ of $M_{2}(F)$.

Proposition 3.1. Let $A$ and $B$ be $F$-algebras such that $B \subseteq A$ and consider $\lambda \vdash n$ a fixed partition. If $\psi\left(I_{\lambda}\right) \cap C(B)=\operatorname{span}_{F}\left\{\psi\left(f_{1}\right), \cdots \psi\left(f_{r}\right)\right\}$ where $f_{j}^{\prime} s$ are of form $\sum_{i=1}^{d} \alpha_{i} e_{i} S_{i k_{j}} \in F S_{n} e_{k_{j}}$ with $k_{j} \in\{1, \ldots, d\}$ and $j \in\{1, \ldots, r\}$ then $\psi\left(I_{\lambda}\right) \cap C(A)=\operatorname{span}_{F}\left\{\psi\left(f_{i_{1}}\right), \ldots, \psi\left(f_{i_{s}}\right)\right\}$ where $i_{1}, \ldots, i_{s} \in\{1, \ldots, r\}$.

Proof. If $\psi(g) \in \psi\left(I_{\lambda}\right) \cap C(A)$ then for all $k=1, \ldots d$ there exist $\psi\left(g_{\lambda}^{k}\right) \in$ $\psi\left(F S_{n} e_{k}\right) \cap C(A) \subseteq \psi\left(F S_{n} e_{k}\right) \cap C(B)$ such that $\psi\left(g_{\lambda}\right)=\psi\left(g_{\lambda}^{1}\right)+\ldots+\psi\left(g_{\lambda}^{d}\right)$.

On the other hand, since $\psi(g) \in \psi\left(I_{\lambda}\right) \cap C(A) \subseteq \psi\left(I_{\lambda}\right) \cap C(B)$ there exist $\beta_{j}^{\prime} s \in F$ such that $\psi\left(g_{\lambda}\right)=\beta_{1} \psi\left(f_{1}\right)+\ldots+\beta_{r} \psi\left(f_{r}\right)$. It implies

$$
\psi\left(g_{\lambda}\right)=\psi\left(\beta_{1} f_{1}\right)+\ldots+\psi\left(\beta_{r} f_{r}\right)=\psi\left(g_{\lambda}^{1}\right)+\ldots+\psi\left(g_{\lambda}^{d}\right) .
$$

Using that $\psi\left(\beta_{j} f_{j}\right) \in \psi\left(F S_{n} e_{k_{j}}\right) \cap C(B)$ by the uniqueness of the decomposition it follows that

$$
\begin{gathered}
\psi\left(g_{\lambda}^{k_{j}}\right)=\psi\left(\beta_{j} f_{j}\right) \text { for all } j \in\{1,2, \ldots, r\} \\
\psi\left(g_{\lambda}^{k}\right)=0 \text { if } k \notin\left\{k_{1}, \ldots, k_{r}\right\} .
\end{gathered}
$$

So $\psi\left(f_{j}\right) \in C(A), \forall j \in\{1, \ldots, r\}$ such that $\beta_{j} \neq 0$ and the result follows.

Now we will see the results which are important to decide whether an element of degree $n$ of type $\mathcal{T}$ of $M_{2}(F)$ is an element of degree $n$ of type $\mathcal{T}$ of $M_{2}(E)$ or not.

We have the following remark from Vishne [13].

Remark 3.2. Let $f \in P_{n}$. Then for all $k \geq 2$ :

$f \equiv 0$ in $M_{k}(E)$ if and only if for any choice of elementary matrices $A_{i}=E_{a_{i} b_{i}}$ and either $v_{i}^{*}=v_{i}$ or $v_{i}^{*}=1$, the substitution $x_{i} \mapsto A_{i} v_{i}^{*}$ in $f$ gives zero.

Next we have an important result to finish our algorithms.

Proposition 3.3. Let $\left\{j_{1}, \cdots, j_{q}\right\} \subset\{1, \cdots, n\}$ with $j_{1}<\cdots<j_{q}$ and $M=M\left(x_{1}, \cdots, x_{n}\right)=\alpha_{\sigma} x_{\sigma(1)} \ldots x_{\sigma(n)}$ a monomial in $P_{n}$. If $r_{1}<\cdots<r_{q}$ such that $\sigma\left(r_{i}\right)=j_{l_{i}}$ where $l_{i} \in\{1, \cdots, q\}$ for all $i \in\{1, \cdots, q\}$ then the 
substitution $x_{i} \mapsto A_{i} v_{i}^{*}$ in the monomial $M$ where $A_{i}$ is an unitary matrix and $v_{i}^{*}=v_{i}$ if $i \in\left\{j_{1}, \cdots, j_{q}\right\}$ and $v_{i}^{*}=1$ if $i \in\{1, \cdots, n\} \backslash\left\{j_{1}, \cdots, j_{q}\right\}$ we have

$$
M\left(A_{1} v_{1}^{*}, \cdots, A_{n} v_{n}^{*}\right)=\left(\operatorname{sgn} \tau_{\sigma}\right) \alpha_{\sigma} A_{\sigma(1)} \ldots A_{\sigma(n)}\left(v_{j_{1}} \ldots v_{j_{q}}\right)
$$

where $\tau_{\sigma} \in S_{q}$ with $\tau_{\sigma}(1)=l_{1}, \cdots, \tau_{\sigma}(q)=l_{q}$.

Proof. In fact the substitution $x_{i} \mapsto A_{i} v_{i}^{*}$ in the monomial $M$ where $v_{i}^{*}=v_{i}$ if $i \in\left\{j_{1}, \ldots, j_{q}\right\}$ and $v_{i}^{*}=1$ if $i \in\{1, \ldots, n\} \backslash\left\{j_{1}, \ldots, j_{q}\right\}$ is the same as $x_{\sigma(t)} \mapsto A_{\sigma(t)} v_{\sigma(t)}^{*}$ for all $t \in\{1, \ldots, n\}$, where $v_{\sigma(t)}^{*}=v_{\sigma(t)}$ if $t \in\left\{r_{1}, \ldots, r_{q}\right\}$ and $v_{\sigma(t)}^{*}=1$ if $t \in\{1, \ldots, n\} \backslash\left\{r_{1}, \ldots, r_{q}\right\}$. Thus

$$
\begin{aligned}
M\left(A_{1} v_{1}^{*}, \ldots, A_{n} v_{n}^{*}\right) & =\alpha_{\sigma} A_{\sigma(1)} v_{\sigma(1)}^{*} \ldots A_{\sigma(n)} v_{\sigma(n)}^{*} \\
& =\alpha_{\sigma} A_{\sigma(1)} \ldots A_{\sigma(n)}\left(v_{\sigma\left(r_{1}\right)} \ldots v_{\sigma\left(r_{q}\right)}\right) \\
& =\alpha_{\sigma} A_{\sigma(1)} \ldots A_{\sigma(n)}\left(v_{j_{\tau_{\sigma}(1)}} \ldots v_{j_{\tau_{\sigma}(q)}}\right) \\
& =\left(\operatorname{sgn} \tau_{\sigma}\right) \alpha_{\sigma} A_{\sigma(1)} \ldots A_{\sigma(n)}\left(v_{j_{1}} \ldots v_{j_{q}}\right) .
\end{aligned}
$$

Now let us explain how to construct elements in $C\left(M_{2}(E)\right)$.

CR "CentralPolynomialTest $(h)$ ": verifies if a polynomial $h\left(x_{1}, \cdots, x_{n}\right)$ belongs to $C\left(M_{2}(E)\right)$ and it works from some steps.

(1) Let

$$
f=f\left(x_{1}, \cdots, x_{n}\right)=\sum_{\sigma \in S_{n}} \alpha_{\sigma} x_{\sigma(1)} \ldots x_{\sigma(n)} \in P_{n} .
$$

We have:

- According to Remark 3.2, $f \in I d\left(M_{2}(E)\right)$ if and only if for any choice $\left\{A_{1} v_{1}^{*}, \cdots, A_{n} v_{n}^{*}\right\}$ where $A_{i}$ 's are elementary matrices and $v_{i}^{*}=v_{i}$ or 1 , we have

$$
f\left(A_{1} v_{1}^{*}, \cdots, A_{n} v_{n}^{*}\right)=0 .
$$

- There exist $4^{n} .2^{n}$ ways to make that choice: $4^{n}$ possibilities to the sequence $\left\{A_{1}, \cdots, A_{n}\right\}$ and $2^{n}$ possibilities to the sequence $v_{1}^{*}, \cdots, v_{n}^{*}$.

- We identify

$$
\begin{aligned}
\left\{v_{1}^{*}, \cdots, v_{n}^{*}\right\} & \leftrightarrow\left\{j_{1}, \cdots, j_{q}\right\} \subseteq\{1, \cdots, n\}, \text { with } j_{1}<\cdots<j_{q} \\
& \text { where } v_{i}^{*}=v_{i} \text { if } i \in\left\{j_{1}, \cdots, j_{q}\right\} \text { and } \\
& v_{i}^{*}=1 \text { if } i \in\{1, \cdots, n\} \backslash\left\{j_{1}, \cdots, j_{q}\right\} .
\end{aligned}
$$


- For all $4^{n} .2^{n}$ choices $\left\{A_{1} v_{1}^{*}, \cdots, A_{n} v_{n}^{*}\right\}$ we have

$$
\begin{gathered}
f\left(A_{1} v_{1}^{*}, \cdots, A_{n} v_{n}^{*}\right)=0 \\
\Leftrightarrow \\
\sum_{\sigma \in S_{n}}\left(\operatorname{sgn} \tau_{\sigma}\right) \alpha_{\sigma} A_{\sigma(1)} \ldots A_{\sigma(n)}\left(v_{j_{1}} \ldots v_{j_{q}}\right)=0 \\
\Leftrightarrow \\
\sum_{\sigma \in S_{n}}\left(\operatorname{sgn} \tau_{\sigma}\right) \alpha_{\sigma} A_{\sigma(1)} \ldots A_{\sigma(n)}=0 .
\end{gathered}
$$

(2) For

$$
h=h\left(x_{1}, \cdots, x_{n}\right)=\sum_{\sigma \in S_{n}} \alpha_{\sigma} x_{\sigma(1)} \ldots x_{\sigma(n)} \in P_{n}
$$

and each sequence $\left\{A_{1}, \cdots, A_{n+1}\right\}$ of elementary matrices and each subset $\left\{j_{1}, \cdots, j_{q}\right\} \subseteq\{1, \cdots, n+1\}$, the routine constructs a new polynomial

$$
f=f\left(x_{1}, \cdots, x_{n}\right)=\left[h, x_{n+1}\right]=\sum_{\gamma \in S_{n+1}} \alpha_{\gamma} x_{\gamma(1)} \ldots x_{\gamma(n+1)} \in P_{n+1} .
$$

(3) For each monomial of $f$ the routine constructs the permutation $\tau_{\gamma}$ as in the Proposition 3.3 and computes the product $A_{\gamma(1)} \ldots A_{\gamma(n)} A_{\gamma(n+1)}$ to form the matrix

$$
A=\sum_{\gamma \in S_{n+1}}\left(\operatorname{sgn} \tau_{\gamma}\right) \alpha_{\gamma} A_{\gamma(1)} \ldots A_{\gamma(n)} A_{\gamma(n+1)} .
$$

(4) If $A=0$ for all sequences $\left\{A_{1}, \cdots, A_{n+1}\right\}$ and all subsets $\left\{j_{1}, \cdots, j_{q}\right\} \subseteq\{1, \cdots, n+1\}$ then $f \in \operatorname{Id}\left(M_{2}(E)\right)$. As a consequence, $h \in C\left(M_{2}(E)\right)$.

To finish, we use a new routine "PolynomialIdentitiesTest $(h)$ " to determine if a polynomial $h \in C\left(M_{2}(E)\right)$ is or not an identity, since our interest is to find non-trivial central polynomials. The idea used in this routine is analogous to that one used in the previous, where the difference consists in considering $h$ instead of $f$.

The implementation of the routines above guaranteed the following.

Theorem 3.4. If $f$ is a non-trivial central polynomial of $M_{2}(E)$ then degree of $f \geq 9$. 


\section{Final remarks}

The procedure was designed to find central polynomials of arbitrary degree of the matrix algebra $M_{k}(E)$, for any $k \geq 2$. The tests done using the software GAP showed that the algebra $M_{2}(E)$ doesn't contain central polynomials of degree 7 . Since Vishne informed the only elements of degree 8 of $C\left(M_{2}(E)\right)$ are the identities, to construct central polynomials of bigger degree, we have to consider $n=9$. The work in this case requires a large number of computations and our next goal is improving the algorithms to finally determine the minimal degree of a non-trivial element in $C\left(M_{2}(E)\right)$. The sources of the computional routines and procedures, together with some examples, are available upon request by e-mail.

\section{References}

[1] S. Bondari, Constructing the polynomial identities and central identities of degree $<9$ of $3 \times 3$ matrices, Linear Algebra and its Applications 258 (1997) 233-249.

[2] V. Drensky, A minimal basis for the identities of a second-order matrix algebra over a field of characteristic 0, Algebra and Logic 20 (1981) 188-194.

[3] V. Drensky, New central polynomials for a matrix algebra, Israel J. Math. 92 (1995) 235-248.

[4] A. Giambruno and M. Zaicev Polynomial identities and asymptotic methods. AMS Mathematical Surveys and Monographs, Vol. 122 - Providence R.I., 2005.

[5] The GAP Group, 2002. GAP - Group, Algorithms and Programming version 4.3. Available from: http://www.gap-system.org

[6] G. James and A. Kerber, The representation theory of the symmetric group. London: Addison-Wesley Publishing Company, 1981.

[7] A. R. Kemer, Varieties and $\mathbb{Z}_{2}$-graded algebras, Math. USSR, Izv. 25 (1985) 359-374.

[8] A. R. Kemer, On the multilinear components of the regular prime varieties, in: Methods in Ring Theory, M. Dekker, New York (1998) 171-183 (Lecture Notes Pure Appl. Math. 138).

[9] D. Krakowski and A. Regev, The polynomial identities of the Grassmann algebra, Trans. Amer. Math. Soc. 181 (1973) 429-438.

[10] S. V. Okhitin, Central polynomial of an algebra of second order matrices, Moskow Univ. Math. Bull. 43 No.3. (1988) 49-51.

[11] A. Popov, Identities of the tensor square of a Grassmann algebra, Algebra and Logic 21 (1982) 296-316.

[12] Yu.P. Razmyslov, Identities of algebras and their representations, Translations Math. Monographs 138, AMS - Providence, RI, 1994.

[13] U. Vishne, Polynomial identities of $M_{2}(G)$, Communications in Algebra 30 (1) (2002) 443-454. 